\title{
Thermally activated diffusion and lattice relaxation in (Si)GeSn materials
}

\author{
Nils von den Driesch $\odot,{ }^{1,2}$ Stephan Wirths, ${ }^{2}$ Rene Troitsch, ${ }^{2}$ Gregor Mussler, ${ }^{2}$ Uwe Breuer $\odot,{ }^{3}$ \\ Oussama Moutanabbir, ${ }^{4}$ Detlev Grützmacher, ${ }^{1,2}$ and Dan Buca ${ }^{2}$ \\ ${ }^{1}$ JARA-Institut Green IT, RWTH Aachen, Sommerfeldstraße 14, 52074 Germany \\ ${ }^{2}$ Peter Grünberg Institute (PGI 9) and JARA-FIT, Forschungszentrum Jülich, 52425 Jülich, Germany \\ ${ }^{3}$ Zentralinstitut für Engineering, Elektronik und Analytik (ZEA 3), Forschungszentrum Jülich, 52425 Jülich, Germany \\ ${ }^{4}$ Department of Engineering Physics, École Polytechnique de Montréal, Montréal, C.P. 6079, \\ Succ. Centre-Ville, Montréal, Québec, Canada H3C $3 A 7$
}

(Received 7 November 2019; revised manuscript received 13 February 2020; accepted 25 February 2020; published 20 March 2020)

\begin{abstract}
Germanium-tin (GeSn) alloys have emerged as a promising material for future optoelectronics, energy harvesting, and nanoelectronics owing to their direct band gap and compatibility with existing Si-based electronics. Yet, their metastability poses significant challenges calling for in-depth investigations of their thermal behavior. With this perspective, this work addresses the interdiffusion processes throughout thermal annealing of pseudomorphic GeSn binary and SiGeSn ternary alloys. In both systems, the initially pseudomorphic layers are relaxed upon annealing exclusively via thermally induced diffusional mass transfer of Sn. Systematic postgrowth annealing experiments reveal enhanced $\mathrm{Sn}$ and $\mathrm{Si}$ diffusion regimes that manifest at temperatures below $650{ }^{\circ} \mathrm{C}$. The amplified low-temperature diffusion and the observation of only subtle differences between binary and ternary hint at the unique metastability of the Si-Ge-Sn material system as the most important driving force for phase separation.
\end{abstract}

DOI: 10.1103/PhysRevMaterials.4.033604

\section{INTRODUCTION}

The Si-Ge-Sn semiconductor system has sparked a great deal of interest among scientists because of its unique properties within group IV materials. The demonstrated fundamental direct band gap of this truly silicon-compatible material can pave the way for numerous new applications in the field of opto- and nanoelectronics [1]. Research concerning GeSnbased light emitters was especially spurred by the demonstration of optically pumped lasing in bulk $[2,3]$ and heterostructure layers $[4,5]$ and may one day lead to the convergence of electronics and photonics circuitry [6,7]. Nevertheless, plenty of obstacles in this inherently metastable material system must be overcome to lay the groundwork for real applications.

One of the main challenges posed by ( $\mathrm{Si}) \mathrm{GeSn}$ alloys is maintaining their structural integrity during thermal treatment in order to preserve their intrinsic material properties. For example, thermal budget must be specifically kept low enough to avoid Sn diffusion out of the material and associated segregation [8]. This physical process yields a phase separation with the GeSn equilibrium phase at a Sn content well below the critical value for a direct band gap, rendering it unsuitable for light-emitting devices. Moreover, thermally activated interdiffusion processes can smear out the interfaces and hence

Published by the American Physical Society under the terms of the Creative Commons Attribution 4.0 International license. Further distribution of this work must maintain attribution to the author(s) and the published article's title, journal citation, and DOI. drastically alter the basic heterostructure properties, such as band offsets or confinement potential.

Despite this critical importance, detailed studies of thermal behavior of metastable $(\mathrm{Si}) \mathrm{GeSn}$ materials are still conspicuously missing in literature. In contrast, an extensive body of knowledge is available on the canonical binary group IV system Si-Ge. In fact, it has been established that-depending on the initial layer strain and dislocation density in the material-both diffusion and strain relaxation via dislocation formation take place upon annealing $[9,10]$ with the former being more relevant in coherent layers. Similarly, the mechanism governing thermal relaxation behavior in $(\mathrm{Si}) \mathrm{GeSn}$ alloys is indistinct, counterintuitive, and not fully understood yet, encompassing Sn diffusion, phase segregation, and defect nucleation [11-15]. The observed rather low thermal stability of GeSn, compared to SiGe alloys, however, originates expectedly from the low solid solubility of $\mathrm{Sn}$ in $\mathrm{Ge}$, making even unstrained layers thermodynamically metastable [16]. This inherent material property is the driving force for low-temperature $\mathrm{Sn}$ diffusion for the formation of Sn-rich precipitates on the surface and nanocrystals inside the material upon annealing, since a phase separation into elemental $\mathrm{Ge}$ and $\beta$-Sn is thermodynamically favored $[11,17,18]$. However, the role of strain in altering the thermodynamic properties of GeSn layers is yet to be determined.

There is even less certainty for the case of ternary SiGeSn alloys. Fundamentally, phase separation in ternary alloys should differ from the binary case, since a larger possible number of intermediate phases can be formed [19]. The solubility of $\mathrm{Sn}$ in a $\mathrm{SiGe}$ matrix is even smaller compared to a Ge one [16], which may point to less-stable ternaries in 


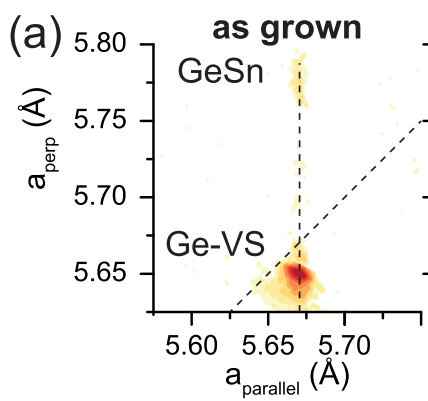

$500^{\circ} \mathrm{C}, 10 \mathrm{~min}$

(b) as grown

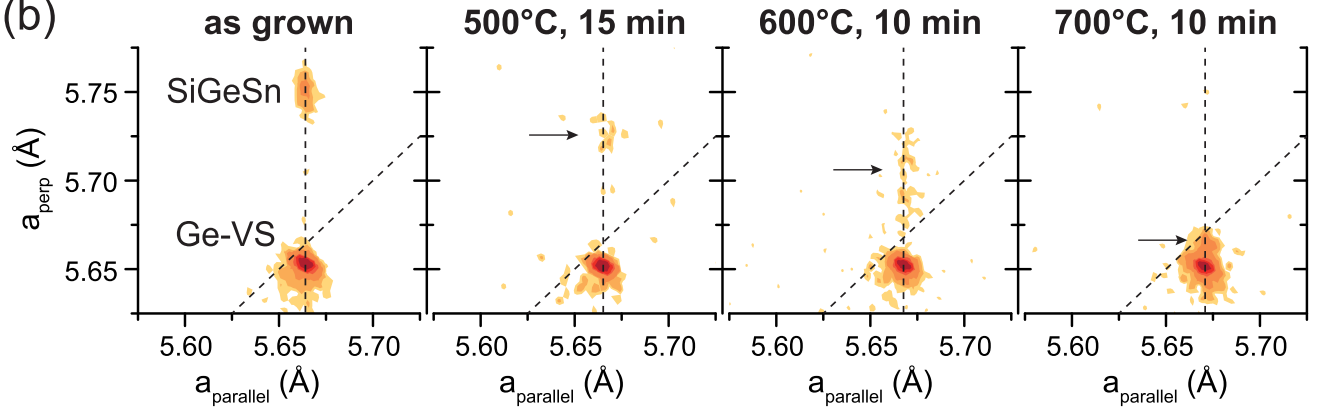

$600^{\circ} \mathrm{C}, 10 \mathrm{~min}$

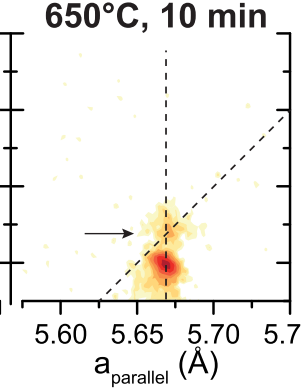

(c)

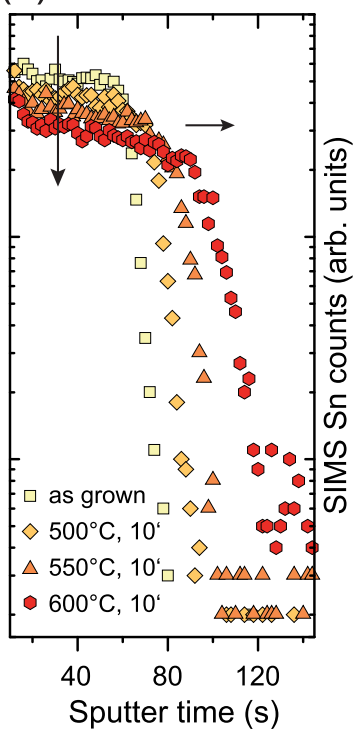

FIG. 1. XRD Reciprocal space maps of coherently grown (a) $\mathrm{Ge}_{0.92} \mathrm{Sn}_{0.08}$ and (b) $\mathrm{Si}_{0.10} \mathrm{Ge}_{0.81} \mathrm{Sn}_{0.09}$ samples, as well as (c) $\mathrm{SIMS}$ spectra of the former, annealed at different temperatures.

that case. On the other hand, however, the enhanced mixing entropy in ternary alloys was proposed to explain a predicted stability enhancement [20].

In this work, we investigate the diffusion and lattice relaxation of both pseudomorphic GeSn binary and SiGeSn ternary alloys upon thermal annealing, to identify the behavior of each one of those systems and reveal possible differences between them.

\section{SAMPLE PREPARATION}

Epitaxial growth of thin films was conducted at temperatures below $400{ }^{\circ} \mathrm{C}$ in a commercial AIXTRON Tricent reduced-pressure chemical vapor deposition reactor employing a reactive gas source epitaxial process using the precursors digermane $\left(\mathrm{Ge}_{2} \mathrm{H}_{6}\right)$, disilane $\left(\mathrm{Si}_{2} \mathrm{H}_{6}\right)$, and tin tetrachloride $\left(\mathrm{SnCl}_{4}\right)$ [21]. Alloys were grown on Ge buffers to minimize the large lattice mismatch to the underlying 200-mm Si(001) wafers. Two pairs of GeSn and SiGeSn samples with matching Sn concentrations of roughly 6 and 9 at. \%, respectively, were prepared to allow a direct comparison between binary and ternary layers. The thickness of all layers was beneath $50 \mathrm{~nm}$, chosen well below the critical thickness for plastic strain relaxation, to suppress the impact of defects on the diffusion experiments. The pair of GeSn and SiGeSn layers, which was intended for determining the diffusion constants, was capped by additional 300 and $150 \mathrm{~nm} \mathrm{Ge}$, respectively. The cap layers were grown at the very same temperature as the binary and ternary to rule out an influence of the sample surface on the diffusion process. Initial material parameters, such as composition and layer thickness, were probed by Rutherford backscattering spectrometry (RBS) and validated by x-ray diffraction (XRD) techniques. RBS channeling measurements, performed on all samples along the (001) crystal direction, affirmed a good crystalline quality of those without misfit dislocations, which could otherwise influence the diffusion behavior.

For the experiments, samples were annealed in an industrial Mattson Helios rapid thermal processing system in a temperature range between 350 and $850{ }^{\circ} \mathrm{C}$ under nitrogen atmosphere for different periods of time between 3 and $30 \mathrm{~min}$.

\section{RESULTS}

To elucidate the thermal behavior of ( $\mathrm{Si}$ )GeSn semiconductors, systematic annealing experiments were performed on $\mathrm{Ge}_{0.92} \mathrm{Sn}_{0.08}$ and $\mathrm{Si}_{0.10} \mathrm{Ge}_{0.81} \mathrm{Sn}_{0.09}$ samples, pseudomorphically grown on top of Ge buffers. The buffer layer exhibits a very low threading dislocation density $\left(<1 \times 10^{7} \mathrm{~cm}^{-2}\right)$ [22], which defines the upper limit of threading arms gliding through the pseudomorphic GeSn layer. Reciprocal space maps (RSM) of the former, obtained from XRD experiments around the asymmetric (224) reflection, are shown for different annealing temperatures in Fig. 1(a). Coherent growth is proven by the matching in-plane lattice constants $a_{\text {parallel }}$ of GeSn layer and Ge virtual substrate (Ge-VS). Annealing of the samples for $30 \mathrm{~min}$ at temperatures below $450{ }^{\circ} \mathrm{C}$ does not have a distinct effect on the layer strain, which is consistent with thermal stability data on alloys with similar composition [11]. Subsequent to thermal annealing at $500{ }^{\circ} \mathrm{C}$ for $10 \mathrm{~min}$, however, the GeSn peak moves towards smaller out-of-plane lattice constants $a_{\text {perp }}$, closer to the Ge-VS lattice. Simultaneously, the in-plane lattice constant remains fixed at the Ge-VS value. Such lattice parameter evolution indicates that, although $\mathrm{Sn}$ diffuses out of the layer, the GeSn layer always remains coherently strained on top of the buffer.

By examining the XRD data recorded on the pseudomorphic $\mathrm{Si}_{0.10} \mathrm{Ge}_{0.81} \mathrm{Sn}_{0.09}$ layers [Fig. 1(b)], the ternary was found to behave qualitatively similarly to the binary case 
(a)

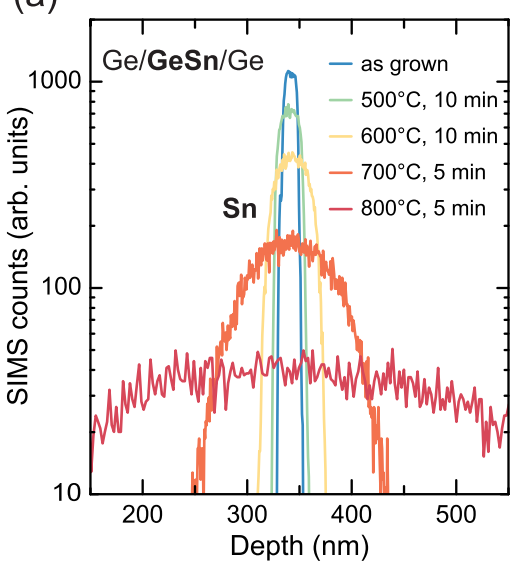

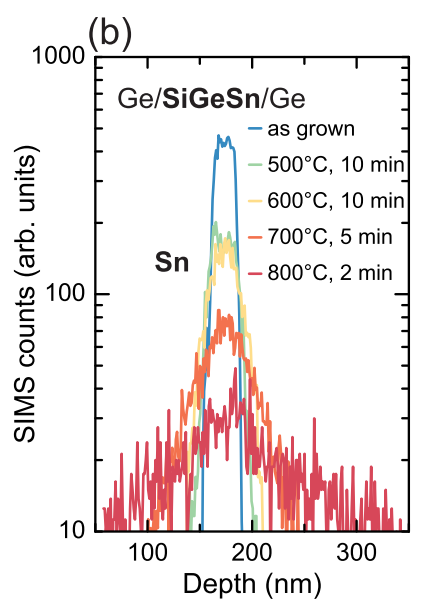

FIG. 2. Sn diffusion in pseudomorphically grown (a) $\mathrm{Ge}_{0.94} \mathrm{Sn}_{0.06}$ and (b) $\mathrm{Si}_{0.040} \mathrm{Ge}_{0.895} \mathrm{Sn}_{0.065}$, sandwiched between two layers of Ge, respectively. (c) Comparison of Sn diffusion coefficients to literature data.

[Fig. 1(a)]. Also, the gradual diffusion of Sn upon annealing causes a shift of the SiGeSn XRD signal towards the buffer, yet remaining a coherently strained layer. In both samples, annealing above $700{ }^{\circ} \mathrm{C}$ decreases the mean $\mathrm{Sn}$ content to 1 at. \%. In such mixture, a thermodynamically stable solid solution is formed, which triggers strongly enhanced Sn diffusion, as we will discuss later on.

Diffusion of $\mathrm{Sn}$ in samples can be easily made visible from secondary ion mass spectroscopy (SIMS) profiles, which are displayed in Fig. 1(c). These measurements unravel the kinetics of Sn diffusion throughout thermal annealing for $10 \mathrm{~min}$ at different temperatures. Besides the expected spread of $\mathrm{Sn}$ in larger depths, some segregation of $\mathrm{Sn}$ is also observed on the surface above $600{ }^{\circ} \mathrm{C}$. Interestingly, the Sn composition profiles themselves remain rather rectangular during annealing. Sn atoms diffuse roughly $40 \mathrm{~nm}$ into the Ge buffer when annealed at $600{ }^{\circ} \mathrm{C}$ for $10 \mathrm{~min}$, yet it only reduces the $\mathrm{Sn}$ concentration of the layer and smears the $\mathrm{GeSn} / \mathrm{Ge}$ interface. No Gaussian shape of the composition profiles, as expected from a composition-independent diffusion coefficient, can be seen in our experiments.

In general, the observed gradual diffusion of $\mathrm{Sn}$ in pseudomorphic layers stands in distinct contrast to the recorded behavior in partially relaxed material. In the latter, thermal annealing leads to an abrupt transition to the two equilibrium phases [11,23-25]. This is linked to a preferential diffusion of Sn atoms along dislocations [26], enhancing the clustering of $\mathrm{Sn}$ atoms that ultimately leads to the formation of $\beta$-Sn crystallites and precipitates on the surface and in bulk. On the other hand, as seen above, Sn diffusion in pseudomorphic layers is a gradual process, depending strongly on temperature and annealing times.

For diffusion experiments, 20- and 25-nm-thin $\mathrm{Ge}_{0.94} \mathrm{Sn}_{0.06}$ and $\mathrm{Si}_{0.040} \mathrm{Ge}_{0.895} \mathrm{Sn}_{0.065}$ samples, respectively, were grown coherently on top of Ge-buffered $\mathrm{Si}(001)$ wafers. To investigate diffusion into the surrounding Ge matrix and suppress surface segregation, which will influence the former as for the samples in Fig. 1, both GeSn and SiGeSn layers were capped in situ with 300- and 150-nm-thick Ge layers, respectively.

Figure 2(a) depicts the diffusion of Sn atoms in the GeSn structure after annealing for different periods between 3 and
$10 \mathrm{~min}$ at temperatures between 500 and $800^{\circ} \mathrm{C}$, as no distinct effect was observed at lower temperatures. In the as-grown sample, Sn atoms exhibit a rather rectangular distribution, hinting at a good GeSn layer quality with sharp interfaces. Up to $600{ }^{\circ} \mathrm{C}$ annealing, the Sn profiles look flat and with steeper sides than one would expect for a constant diffusion coefficient. Annealing above $600{ }^{\circ} \mathrm{C}$ transforms the profiles into a more Gaussian-like shape. Thereupon, Sn content in the layer is strongly reduced-more than a factor of 2-after annealing at $600{ }^{\circ} \mathrm{C}$ for $10 \mathrm{~min}$ compared to the as-grown case. A qualitatively similar behavior can be seen for $\mathrm{Sn}$ diffusion in a SiGeSn ternary, as shown in Fig. 2(b), indicating an increased $\mathrm{Sn}$ interdiffusion into the surrounding Ge. In comparison to the binary, however, Sn diffusion in SiGeSn sets in relatively easier, as the $\mathrm{Sn}$ peak concentration decreases more rapidly at lower temperatures.

The distinct diffusion behavior of Sn in the binary and ternary $(\mathrm{Si}) \mathrm{GeSn}$ materials are summarized in Fig. 2(c). In this figure, Sn diffusion coefficients, determined by fitting appropriate solutions of the one-dimensional diffusion equation [27], are plotted against $\left(k_{B} T\right)^{-1}$ in a large temperature range between 500 and $850{ }^{\circ} \mathrm{C}$. Low- and high-temperature regimes can clearly be distinguished from varying slopes in the $\mathrm{Ar}$ rhenius plot. Above $\sim 650{ }^{\circ} \mathrm{C}$, diffusion of $\mathrm{Sn}$ atoms from $\mathrm{GeSn}$, as well as $\mathrm{SiGeSn}$, is similar to reports on diffusion of implanted Sn into Ge wafers [28-30], as indicated by lines in Fig. 2(c). The temperature-dependent behavior of the $\mathrm{Sn}$ diffusion coefficient in this region $D(T)$ can be described by an Arrhenius behavior

$$
D(T)=D_{0} \times \exp \left(-\frac{E_{A}}{k_{B} T}\right),
$$

with a constant preexponential factor $D_{0}$, activation energy $E_{A}$, and Boltzmann constant $k_{B}$. An overview on the obtained parameters can be found in Table I. The activation energies of $3.10 \pm 0.07 \mathrm{eV}(\mathrm{GeSn})$ and $2.87 \pm 0.03 \mathrm{eV}(\mathrm{SiGeSn})$ are still in line with literature values, where a spread between 3.26 and $2.90 \mathrm{eV}$ has been reported for ion-implanted $\mathrm{Sn}$ impurities in Ge [28-30]. A slightly smaller activation energy in the case of SiGeSn may indicate a larger number of vacancies in that material, as we will discuss in the upcoming section. 
TABLE I. Overview of determined activation energies $E_{\mathrm{A}}$ and preexponential factors $D_{0}$ for diffusion of $\mathrm{Sn}$ and $\mathrm{Si}$ from GeSn and $\mathrm{SiGeSn}$ layers in the high- and low-temperature regimes.

\begin{tabular}{|c|c|c|c|c|c|}
\hline \multirow[b]{2}{*}{ Regime } & \multirow[b]{2}{*}{ Material stack } & \multicolumn{2}{|c|}{$\mathrm{Sn}$} & \multicolumn{2}{|c|}{$\mathrm{Si}$} \\
\hline & & $E_{\mathrm{A}}(\mathrm{eV})$ & $D_{0}\left(\mathrm{~cm}^{2} / \mathrm{s}\right)$ & $E_{\mathrm{A}}(\mathrm{eV})$ & $D_{0}\left(\mathrm{~cm}^{2} / \mathrm{s}\right)$ \\
\hline High $T$ & $\mathrm{Ge} / \mathrm{GeSn} / \mathrm{Ge}$ & $3.10 \pm 0.07$ & $264 \pm 220$ & & \\
\hline$\left(650-850^{\circ} \mathrm{C}\right)$ & $\mathrm{Ge} / \mathrm{SiGeSn} / \mathrm{Ge}$ & $2.87 \pm 0.03$ & $12 \pm 5$ & $3.27 \pm 0.03$ & $31 \pm 9$ \\
\hline Low $\mathrm{T}$ & $\mathrm{Ge} / \mathrm{GeSn} / \mathrm{Ge}$ & $1.81 \pm 0.09$ & & & \\
\hline$\left(500-650{ }^{\circ} \mathrm{C}\right)$ & $\mathrm{Ge} / \mathrm{SiGeSn} / \mathrm{Ge}$ & $1.13 \pm 0.11$ & & $0.50 \pm 0.10$ & \\
\hline
\end{tabular}

The overall diffusion coefficient above $650{ }^{\circ} \mathrm{C}$, however, is always slightly smaller than in GeSn. Likely the presence and diffusion of $\mathrm{Si}$ atoms, which also occurs by a vacancymediated mechanism [31], impacts diffusion of the second species and leads to diverging activation energies.

In the low-temperature regime below $\sim 650{ }^{\circ} \mathrm{C}$ an interesting change in diffusion behavior becomes apparent. The temperature dependence is less pronounced and cannot be described by the same activation energies anymore. Reduced activation energies of $1.81 \pm 0.09 \mathrm{eV}(\mathrm{GeSn})$ and $1.13 \pm$ $0.10 \mathrm{eV}(\mathrm{SiGeSn})$ indicate strongly enhanced diffusion in that regime. Such enhancement may be predominantly driven by different physical origins, such as the concentration gradient or strain. Importantly, those can manifest in the thermal behavior only due to the absence of dislocations, which would otherwise overshadow their effects. The individual impact of those driving forces will be reconsidered in the Discussion section.

(a)

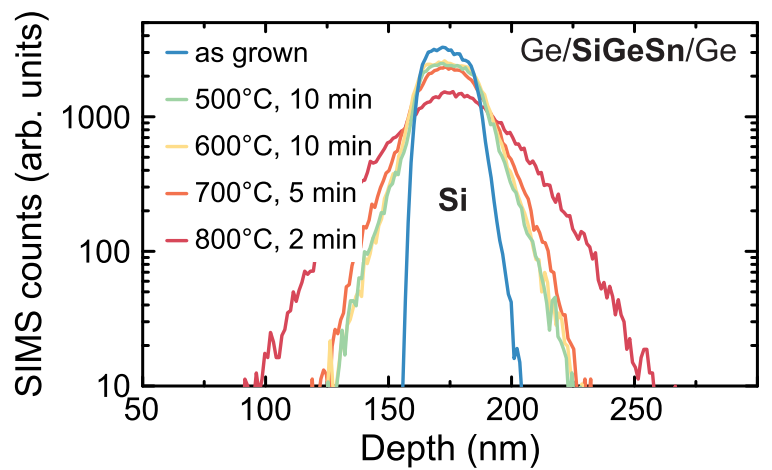

(b)

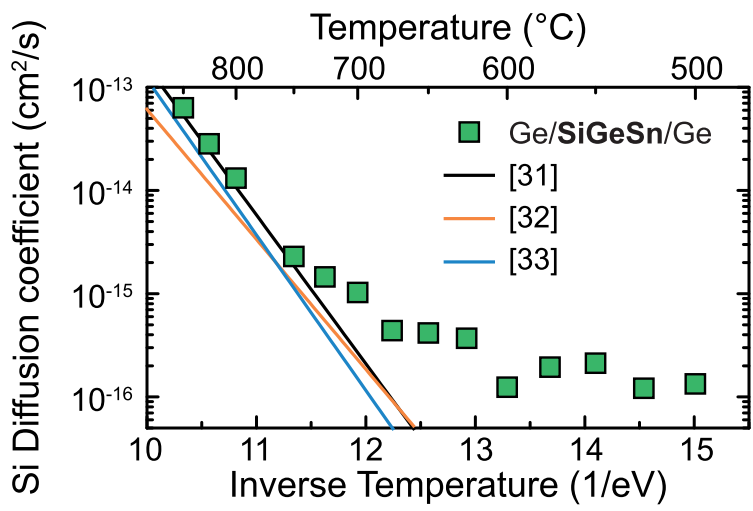

FIG. 3. (a) Si diffusion from $\mathrm{Si}_{0.040} \mathrm{Ge}_{0.895} \mathrm{Sn}_{0.065}$ and (b) comparison of the derived diffusion coefficients to literature data.
Diffusion of $\mathrm{Si}$ atoms, depicted in Fig. 3(a), shows a similar behavior compared to Sn diffusion. Again, it consists of two different regimes above and below $\sim 700{ }^{\circ} \mathrm{C}$, as demonstrated by the diffusion coefficients in Fig. 3(b). This observation is rather surprising, since previous works concerning Si diffusion in Ge always found a behavior well described by a single activation energy down to $650{ }^{\circ} \mathrm{C}$ [32] Indeed, the determined activation energy of $3.27 \pm 0.03 \mathrm{eV}$ in the high-temperature regime very well matches literature values of vacancy-mediated Si diffusion in Ge, which spread between 2.90 and $3.47 \mathrm{eV}$ [31-33]. The larger activation energy compared to $\mathrm{Sn}$ diffusion is typically attributed to a size effect. The smaller $\mathrm{Si}$ atom size results in a reduced attractive interaction with vacancies, which determine diffusion behavior of group IV elements in Ge lattices [31]. In the low-temperature regime, the activation energy drops down to $0.50 \mathrm{eV}$.

\section{DISCUSSION}

An important observation in our experiments is that only isolated Sn diffusion occurs during thermal annealing in both binary and ternary alloys. In literature, also strain relaxation upon thermal annealing via defect formation was observed for both initially relaxed GeSn material [34], as well as for coherently strained GeSn films on rather thin Ge buffer layers $[14,35]$. We attribute the different behavior upon annealing to differences in initial crystallinity and buffer quality. In case of low initial threading dislocation densities (TDDs), as it is the case for our coherently grown material, the formation of isolated defects is energetically not favored. Thus, thermal strain relaxation occurs rather elastically via diffusion of $\mathrm{Sn}$ atoms, as demonstrated by RSM of the annealed samples in Fig. 1. Grown-in dislocations, on the other hand, are present in partly relaxed $\mathrm{GeSn}$ or coherently grown material on top of Ge buffers with larger TDDs. In those, multiplication and gliding of preexisting dislocations are kinetically enabled during thermal annealing. This mechanism generates an array of strain-relieving misfit segments, which is well known from relaxation via dislocations in SiGe alloys [36]. Furthermore, the generation of dislocations also changes the observed phase separation behavior of GeSn alloys, which is part of an ongoing investigation that will be reported elsewhere.

Generally, diffusion in a multicomponent system is driven by a minimization of the Gibbs free energy. Therefore, concentration gradients between the solute and the surrounding matrix will strongly impact interdiffusion, which was shown for example in the Si-Ge system $[37,38]$. On the other hand, also strain inside the material may enhance diffusion towards 
an equilibrium state via mass transfer, as indicated in literature $[10,39]$. Additionally, the thermodynamic instability of the (Si-)Ge-Sn material system may also enhance $\mathrm{Sn}$ diffusion out of the binary, as it moves the system closer to its thermodynamic equilibrium composition (below $\sim 1$ at. \% Sn) [40].

The emergence of two clearly separated temperature regimes of the Sn diffusion coefficient in our work is in stark contrast to previous studies reported in literature. We attribute the presence of the low-temperature regime to the metastability of the $\mathrm{Si}-\mathrm{Ge}-\mathrm{Sn}$ material system, which is unique among the group IV alloys. Above the transition temperature of roughly $650{ }^{\circ} \mathrm{C}$ [see Fig. 2(c)], the remaining (mean) Sn concentrations in the alloys approach the $\mathrm{Sn}$ solid solubility limit of 1 at. \% [cf. Figs. 1(a) and 1(b)]. Accordingly, the extracted activation energies for $\mathrm{Sn}$ diffusion match those previously reported for impurity diffusion in a Ge host lattice [28-30].

In the low-temperature regime, however, different physical effects are possible origins of the enhanced diffusion. Material strain, for example, is known to reduce the formation energy of point defects, which mediate diffusion processes. In case of compressively strained $\mathrm{SiGe}$, the formation energy of vacancies is decreased, leading to enhanced Ge interdiffusion [39]. Therefore, compressive strain will likely also have an impact on vacancy-mediated $\mathrm{Sn}$ diffusion in the lowtemperature regimes, but cannot be the sole explanation. Since our investigated $\mathrm{Ge}_{0.94} \mathrm{Sn}_{0.06}$ and $\mathrm{Si}_{0.040} \mathrm{Ge}_{0.895} \mathrm{Sn}_{0.065}$ thin films share the same compressive strain values $(-0.90$ and $-0.89 \%$, respectively), the activation energy reduction should be similar. Instead, the reduction is much more pronounced for the ternary (2.87 to $1.13 \mathrm{eV})$ compared to the binary case $(3.10$ to $1.81 \mathrm{eV})$. Therefore, the metastability of Sn-based alloys, i.e., the gradient in chemical potential, is the main driving force, which triggers the diffusion enhancement in both binary and ternary. Since the solubility of $\mathrm{Sn}$ inside $\mathrm{SiGe}$ is even smaller than in elemental Ge-making SiGeSn ternaries even more metastable alloys [16]-the chemical potential gradient can also explain the observed deviations between binary and ternary, namely reduced activation energies for the latter case.

The drop of activation energy for $\mathrm{Si}$ diffusion in the low-temperature regime, which has never been reported for diffusion in an elemental Ge matrix, may also be linked to the presence of Sn atoms. From atomistic-level analysis it is known that a repulsive interaction between $\mathrm{Si}$ and $\mathrm{Sn}$ atoms is present in ternary $\mathrm{SiGeSn}$ alloys, leading to deviations from a random distribution [41]. This additional effect may influence $\mathrm{Si}$ diffusion in the early stages, before a greater dilution of $\mathrm{Si}$ atoms is reached at higher temperatures.

Another factor, the role of which is not fully understood yet, is the expected presence of vacancies inside (Si)GeSn alloys. Those are inherently present due to the low-temperature epitaxy of thin films and have recently been directly observed using positron annihilation in GeSn alloys [42]. Since vacancies can locally relax the strain surrounding the larger-size $\mathrm{Sn}$ atoms, an attractive interaction between $\mathrm{Sn}$ atoms and vacancies is expected in GeSn alloys; however, the impact of Si has not yet been investigated. Therefore, little is known about differences in formation and migration energies of vacancies between $\mathrm{GeSn}$ and $\mathrm{SiGeSn}$ alloys, which thus cannot be ruled out as possible origin for the different diffusive behavior.

\section{CONCLUSIONS}

In this work, we investigated the effect of thermal annealing on pseudomorphic GeSn binary and SiGeSn ternary alloys. In the absence of dislocations, strain is relaxed only via thermally activated interdiffusion of Sn atoms. The qualitatively same behavior for binaries and ternaries was found in systematic annealing experiments, revealing an additional strongly enhanced $\mathrm{Sn}$ diffusion regime at low temperatures $\left(<650{ }^{\circ} \mathrm{C}\right)$. Similar behavior, albeit less pronounced, was found for diffusion of $\mathrm{Si}$ atoms, pinpointing the unique metastability of the group IV Si-Ge-Sn material system as the most important driving force for interdiffusion. Our results underline the importance of carefully handling the thermal budget of $(\mathrm{Si}) \mathrm{GeSn}$ layers to maintain their pristine material properties during (thermal) processing in order to obtain highly efficient group IV-based opto- and nano electronic devices.

\section{ACKNOWLEDGMENTS}

This research was supported by the German Federal Ministry of Education and Research $(\mathrm{BMBF})$ in frame of the M-ERA.NET project GESNAPHOTO, and the German Research Foundation (DFG) through the project SiGeSn Laser for Silicon Photonics.
[1] S. Wirths, R. Geiger, N. von den Driesch, G. Mussier, T. Stoica, S. Mantl, Z. Ikonić, M. Luysberg, S. Chiussi, J.-M. Hartmann et al., Nat. Photon. 9, 88 (2015).

[2] V. Reboud, A. Gassenq, N. Pauc, J. Aubin, L. Milord, Q.-M. Thai, M. Bertrand, K. Guilloy, D. Rouchon, J. Rothman et al., Appl. Phys. Lett. 111, 092101 (2017).

[3] W. Dou, Y. Zhou, J. Margetis, S. A. Ghetmiri, S. Al-Kabi, W. Du, J. Liu, G. Sun, R. A. Soref, J. Tolle et al., Opt. Lett. 43, 4558 (2018).

[4] N. von den Driesch, D. Stange, D. Rainko, I. Povstugar, P. Zaumseil, G. Capellini, T. Schröder, T. Denneulin, Z. Ikonić, J.-M. Hartmann et al., Adv. Sci. 5, 1700955 (2018).
[5] D. Stange, N. von den Driesch, T. Zabel, F. Armand Pilon, D. Rainko, B. Marzban, P. Zaumseil, J.-M. Hartmann, Z. Ikonić, G. Capellini et al., ACS Photonics 5, 4628 (2018).

[6] L. Vivien, Nature (London) 528, 483 (2015).

[7] A. H. Atabaki, S. Moazeni, F. Pavanello, H. Gevorgyan, J. Notaros, L. Alloatti, M. T. Wade, C. Sun, S. A. Kruger, H. Meng et al., Nature (London) 556, 349 (2018).

[8] C. Schulte-Braucks, N. von den Driesch, S. Glass, A. T. Tiedemann, U. Breuer, A. Besmehn, J.-M. Hartmann, Z. Ikonić, Q.-T. Zhao, S. Mantl et al., ACS Appl. Mater. Interfaces 8, 13133 (2016). 
[9] H. Trinkaus, D. Buca, R. A. Minamisawa, B. Holländer, M. Luysberg, and S. Mantl, J. Appl. Phys. 111, 014904 (2012).

[10] S. S. Iyer and F. K. LeGoues, J. Appl. Phys. 65, 4693 (1989).

[11] P. Zaumseil, Y. Hou, M. A. Schubert, N. von den Driesch, D. Stange, D. Rainko, M. Virgilio, D. Buca, and G. Capellini, APL Mater. 6, 076108 (2018).

[12] C. M. Comrie, C. B. Mtshali, P. T. Sechogela, N. M. Santos, K. van Stiphout, R. Loo, W. Vandervorst, and A. Vantomme, J. Appl. Phys. 120, 145303 (2016).

[13] J.-H. Fournier-Lupien, D. Chagnon, P. Levesque, A. A. AlMutairi, S. Wirths, E. Pippel, G. Mussler, J.-M. Hartmann, S. Mantl, D. Buca et al., ECS Trans. 64, 903 (2014).

[14] C. Chang, H. Li, T.-P. Chen, W.-K. Tseng, H. H. Cheng, C.-T. Ko, C.-Y. Hsieh, M.-J. Chen, and G. Sun, Thin Solid Films 593, 40 (2015).

[15] R. Chen, Y.-C. Huang, S. Gupta, A. C. Lin, E. Sanchez, Y. Kim, K. C. Saraswat, T. I. Kamins, and J. S. Harris, J. Cryst. Growth 365, 29 (2013).

[16] J. P. Fleurial and A. Borshchevsky, J. Electrochem. Soc. 137, 2928 (1990).

[17] A. A. Tonkikh, N. D. Zakharov, A. A. Suvorova, C. Eisenschmidt, J. Schilling, and P. Werner, Cryst. Growth Des. 14, 1617 (2014).

[18] S. Takeuchi, A. Sakai, O. Nakatsuka, M. Ogawa, and S. Zaima, Thin Solid Films 517, 159 (2008).

[19] D. J. Eyre, SIAM J. Appl. Math. 53, 1686 (1993).

[20] J. Xie, A. V. G. Chizmeshya, J. Tolle, V. R. D'Costa, J. Menéndez, and J. Kouvetakis, Chem. Mater. 22, 3779 (2010).

[21] N. von den Driesch, D. Stange, S. Wirths, G. Mussler, B. Holländer, Z. Ikonić, J.-M. Hartmann, T. Stoica, S. Mantl, D. Grützmacher et al., Chem. Mater. 27, 4693 (2015).

[22] J.-M. Hartmann, A. Abbadie, N. Cherkashin, H. Grampeix, and L. Clavelier, Semicond. Sci. Technol. 24, 055002 (2009).

[23] H. Groiss, M. Glaser, M. Schatzl, M. Brehm, D. Gerthsen, D. Roth, P. Bauer, and F. Schäffler, Sci. Rep. 7, 16114 (2017).
[24] O. Gurdal, P. Desjardins, J. R. A. Carlsson, N. Taylor, H. H. Radamson, J.-E. Sundgren, and J. E. Greene, J. Appl. Phys. 83, 162 (1998).

[25] W. Wang, L. Li, Q. Zhou, J. Pan, Z. Zhang, E. S. Tok, and Y. C. Yeo, Appl. Surf. Sci. 321, 240 (2014).

[26] E. V. Dobrokhotov, Phys. Solid State 47, 2257 (2005).

[27] J. Crank, The Mathematics of Diffusion, 2nd ed. (Oxford University Press, Oxford, 1980).

[28] I. Riihimäki, A. Virtanen, S. Rinta-Anttila, P. Pusa, and J. Räisänen, Appl. Phys. Lett. 91, 091922 (2007).

[29] P. Kringhøj and R. G. Elliman, Appl. Phys. Lett. 65, 324 (1994).

[30] M. Friesel, U. Södervall, and W. Gust, J. Appl. Phys. 78, 5351 (1995).

[31] H. H. Silvestri, H. Bracht, J. Lundsgaard Hansen, A. Nylandsted Larsen, and E. E. Haller, Semicond. Sci. Technol. 21, 758 (2006).

[32] J. Räisänen, J. Hirvonen, and A. Anttila, Solid. State. Electron. 24, 333 (1981).

[33] U. Södervall and M. Friesel, Defect Diffus. Forum 143-147, 1053 (1997).

[34] T. Asano, Y. Shimura, N. Taoka, O. Nakatsuka, and S. Zaima, Solid. State. Electron. 83, 71 (2013).

[35] H. Li, Y. X. Cui, K. Y. Wu, W. K. Tseng, H. H. Cheng, and H. Chen, Appl. Phys. Lett. 102, 251907 (2013).

[36] P. M. Mooney, Mater. Sci. Eng. R: Rep. 17, 105 (1996).

[37] B. Holländer, R. Butz, and S. Mantl, Phys. Rev. B 46, 6975 (1992).

[38] W. P. Gillin and D. J. Dunstan, Phys. Rev. B 50, 7495 (1994).

[39] N. E. B. Cowern, P. C. Zalm, P. van der Sluis, D. J. Gravesteijn, and W. B. de Boer, Phys. Rev. Lett. 72, 2585 (1994).

[40] J. W. Cahn and J. E. Hilliard, J. Chem. Phys. 28, 258 (1958).

[41] S. Mukherjee, N. Kodali, D. Isheim, S. Wirths, J. M. Hartmann, D. Buca, D. N. Seidman, and O. Moutanabbir, Phys. Rev. B 95, 161402(R) (2017).

[42] S. Assali, M. Elsayed, J. Nicolas, M. O. Liedke, A. Wagner, M. Butterling, R. Krause-Rehberg, and O. Moutanabbir, Appl. Phys. Lett. 114, 251907 (2019). 